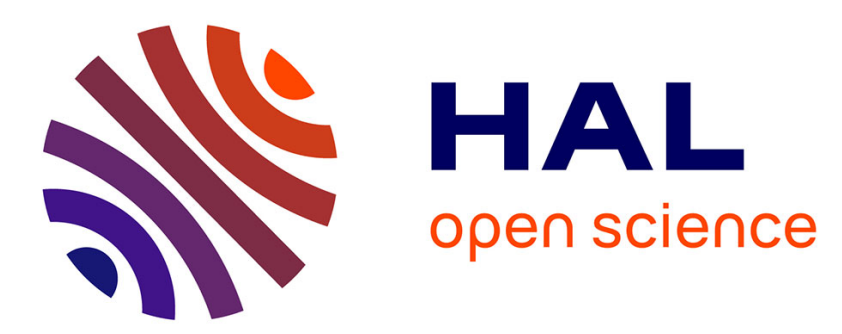

\title{
Tumor growth model of ductal carcinoma: from in situ phase to stroma invasion
}

Olivier Gallinato, Thierry Colin, Olivier Saut, Clair Poignard

\section{To cite this version:}

Olivier Gallinato, Thierry Colin, Olivier Saut, Clair Poignard. Tumor growth model of ductal carcinoma: from in situ phase to stroma invasion. Journal of Theoretical Biology, 2017, 429, pp.253 - 266. 10.1016/j.jtbi.2017.06.022 . hal-01598837

\section{HAL Id: hal-01598837 https://hal.inria.fr/hal-01598837}

Submitted on 5 Oct 2017

HAL is a multi-disciplinary open access archive for the deposit and dissemination of scientific research documents, whether they are published or not. The documents may come from teaching and research institutions in France or abroad, or from public or private research centers.
L'archive ouverte pluridisciplinaire HAL, est destinée au dépôt et à la diffusion de documents scientifiques de niveau recherche, publiés ou non, émanant des établissements d'enseignement et de recherche français ou étrangers, des laboratoires publics ou privés. 


\title{
TUMOR GROWTH MODEL OF DUCTAL CARCINOMA: FROM IN SITU PHASE TO STROMA INVASION
}

\author{
OLIVIER GALLINATO, THIERRY COLIN, OLIVIER SAUT, CLAIR POIGNARD \\ INRIA BORDEAUX-SUD-OUEST, CNRS UMR 5251 \& UNIVERSITÉ DE BORDEAUX \\ 351 COURS DE LA LIBÉRATION, 33405 TALENCE CEDEX, FRANCE
}

\begin{abstract}
This paper aims at modeling breast cancer transition from the in situ stage -when the tumor is confined to the duct- to the invasive phase. Such a transition occurs thanks to the degradation of the duct membrane under the action of specific enzymes so-called matrix metalloproteinases (MMPs). The model consists of advection-reaction equations that hold in the duct and in the surrounding tissue, in order to describe the proliferation and the necrosis of the cancer cells in each subdomain. The divergence of the velocity is given by the increase of the cell densities. Darcy law is imposed in order to close the system. The key-point of the modeling lies in the description of the transmission conditions across the duct. Nonlinear Kedem-Katchalsky transmission conditions across the membrane describe the discontinuity of the pressure as a linear function of the flux. These transmission conditions make it possible to describe the transition from the in situ stage to the invasive phase at the macroscopic level. More precisely, the membrane permeability increases with respect to the local concentration of MMPs. The cancer cells are no more confined to the duct and the tumor invades the surrounding tissue. The model is enriched by the description of nutrients concentration, tumor necrosis factors, and MMPs production. The mathematical model is implemented in a $3 \mathrm{D} \mathrm{C} \mathrm{C}^{++}$-code, which is based on well-adapted finite difference schemes on Cartesian grid. The membrane interface is described by a level-set, and the transmission conditions are precisely approached at the second order thanks to well-suited sharp stencils. Our continuous approach provides new significant insights in the macroscopic modeling of the breast cancer phase transition, due to the membrane degradation by MMP enzymes.
\end{abstract}

\section{INTRODUCTION}

This paper deals with the tumor growth modeling of two types of ductal carcinomas, which are among the most common breast cancers: the ductal carcinoma in situ (DCIS) and the invasive ductal carcinoma (IDC). At the early stage, DCIS remains confined to the duct, thanks to the surrounding membrane, which yields a nonpermeant barrier. Mutated cancer cells can however produce specific enzymes so-called matrix metalloproteinases (MMPs), which degrade the duct membrane. DCIS then turns into an IDC, which is much more agressive. It invades the surrounding healthy tissue and swarms throughout the body, leading to a metastasized breast cancer. The aim of the study is to derive the bases of the macroscopic deterministic modeling of this transition. More precisely partial differential equations, which account for the main biological features of the cancer evolution are proposed. In particular the focus is drawn on the cancer transition from the DCIS phase to IDC under the action of degrading MMP enzymes.

Corresponding author: clair.poignard@inria.fr. 
The model describes the early stage in situ tumor growth, the production of MMPs due to cell hypoxia and then the membrane degradation by MMPs which leads to the tumor invasion of the surrounding stroma. Such a degradation is accounted for thanks to the use of Kedem-Katchalsky transmission conditions, which link the jump of the pressure across the membrane -due to the cell proliferation in the duct- to its normal flux. The influence of the immun $\rrbracket^{1}$ cell located in the stroma on the production of tumor necrosis factors (TNF) and MMPs is also described.

The main insight of the paper is to describe the transition from DCIS into IDC at the continuous macroscopic scale. In particular, we provide a macroscopic tumor growth model that links the production of MMPs to the porosity of the duct membrane.We compare our numerical results with existing biopsy images. Interestingly, our model provides somehow a continuous macroscopic approach of the agent-based model of Kim et al. [20]. The numerical simulations are performed in 3D. The code is based on the finite difference method on Cartesian grid. Level-set method is used to describe the duct membrane. Specific well-adapted stencils are used to describe the KedemKatchalsky transmission conditions along the duct membrane. The overall method is first order accurate in time and space.

1.1. Biological background. Breast consists essentially of milk ducts, lobes and fatty tissue [31]. The ducts are surrounded by a connective tissue, the stroma. They are conmpsed of the basement membrane and of the epithelium surrounding the central opening (called lumen), which is filled with fluid. Ductal carcinoma derives from a dysfunction of the epithelial cells that proliferate uncontrollably. Its origin and the subsequent developments combine genetic and environmental factors, including many underlying mechanisms, which still remain poorly understood. The development of a precancerous lesion in the epithelium, as hyperplasia, increases the risk of abnormalities. The disruptions of the interactions with the membrane can cause an accumulation of abnormal events in the epithelial tissue leading to genetic instabilities [39, 2]. Among the consequences of genetic mutations, the increase in mitosis rate, the loss of cell polarity, the breaks of adhesion junctions and the ability to evade apoptosis lead to uncontrolled cell proliferation and to the tumor growth inside the duct.

As long as the tumor remains confined to the duct, carcinoma is called in situ. The key point is then to determine whether or not (and when) the tumor is able to destroy the membrane and to invade the surrounding tissue, passing from a low-grade cancer, rather non-aggressive, to a high-grade invasive cancer, leading to metastases spreading in the whole organism. The membrane degradation results from the production of MMP enzymes. These enzymes have the property to degrade the collagen fibers, which make up the extracellular matrix (ECM) of biological tissues and membranes. In the case of ductal carcinomas, MMPs degrade locally the membrane, which then becomes porous, leading to the cancer cell invasion. Thus the production of MMPs is the biological feature of the transition from IDCS to IDC [7, 30].

\subsection{Discrete and continuous cancer models.}

${ }^{1}$ TNF are cytokines that are mainly produced by macrophages, but many other cells can produce them, such as CD4+, neutrophils, natural killer cells [17, 38. So we generically consider here that TNF are produced by immune cells. 
Many continuous or discrete models of breast tumor growth have been derived since a decade, however most of them are devoted to DCIS [10, 27, 26], without focusing on the transition from DCIS to IDC. Recently, a modeling based on the evolutionary game theory has been developed by Sartakhti et al. 35] to describe the specific aspects of the complex MMP dynamics. Kim et al proposed a hybrid discrete modeling to study the tumor/stroma interactions [21, 20] at the mesoscale. Accounting for complex chemical and mechanical interactions at both molecular and cell scales, their discrete model reproduces the DCIS-IDC transition. Such modeling works can mimic in vitro experiments and provide interesting features on molecular and cell interactions. However the computational limitations - only a few hundreds of cells can be accounted for in the simulations- and the high variability of cell behavior prevent their calibrations on in vivo and clinical data.

The purpose of the present paper is to provide a continuous macroscopic modeling of the transition from in situ ductal carcinoma to the invasive cancer. We derive here a continuous model, based on partial differential equations, in the same vein as in Colin, Ribba, Saut and colleagues [3, 4, 33, 24]. The motion and the proliferation of cell densities are described thanks to advection-reaction equations. The divergence of the velocity field is given by the total increase of volume due to cell proliferation. Darcy law ensures the closure of the system. The main feature of the present model is the use Kedem-Katchalsky type transmission conditions [22, 31] in order to describe the pressure discontinuity across the duct membrane.

1.3. Ductal carcinoma continuous models in the literature. In its global form, our model is closely related to the model proposed by Franks, Byrne et al. [10, 11]. Advection-reaction equations are used to describe the tumor growth, and the divergence of the velocity field equals the proliferation. The closure of the system is different, since we use a simple Darcy law instead of the Stokes equation used by these authors. Darcy law in tumor growth model was initially proposed by Greenspan et al. [14]. Since our preliminary numerical experiments did not exhibit dramatic different behaviors between Darcy and Stokes equations, we only present here the Darcy law.

The main insight of our paper, compared to the seminal works of Franks, Byrne et al. lies in the derivation of appropriate transmission conditions that makes it possible to account for the tumor invasion. More precisely, the articles of Franks, Byrne et al. focused on the comedo ductal carninoma, a breast cancer confined to the duct [11]. They consider an elastic duct membrane, which confines the tumor to the duct. Free boundary conditions are imposed in order to account for the tumor growth and the elastic deformation. Our point of view is different in the sense that we assume the duct membrane as a rigid domain, but its permeability - that is its capability to confine the tumor to the duct- depends on the production of MMPs enzymes. Therefore the tumor can cross the duct membrane and invade the surrounding tissue. For the sake of simplicity, in the following our membrane is assumed rigid but similarly to Franks, Byrne et al. one can impose free boundary conditions to account for the elastic deformation. This complexification does not change the philosophy of our modeling but it raises technical and numerical difficulties. Thus in this article we focus on the case of a rigid membrane of the duct.

1.4. Outline of the paper. The aim of the paper is to build a continuous model that reproduces qualitatively the different configurations observed in the biopsy observations. We emphasize that 
we aim at paving the bases of the modeling of breast cancer transition. The quantitative calibration with data of well-designed experiments is far beyond the scope of this paper. More precisely, we aim to provide a continuous deterministic model that describes the 3 following phenomena:

- The first-step of the breast cancer growth confined to the duct.

- The influence of tumor necrosis factors and nutrients deprivation on the emergence of a necrotic core.

- The membrane degradation under the action of MMP enzymes, and the subsequent cancer invasion in the stroma.

Our modeling is based upon the following well-established hypotheses [7, 30, 21, 20, 39, 2 ]

- In its early stage, breast cancer is confined to the duct and the tumor is mainly composed of proliferating and necrotic cells. The proportion of invasive metastatic cells is still negligible.

- MT1-MMP enzymes are produced by the cancer cells. However they have to be activated by pro-MMPs produced by the immune cells of the stroma. When activated, such enzymes degrade the duct membrane.

- TNF are mainly produced by macrophages. According to the literature, they paradoxically promote and prevent the tumor necrosis and the cell proliferation, depending on their concentration and on the tumor particularities.

In Section 2, we present our deterministic macroscale model of ductal carcinoma. The core model provides advection equations for cell species, with a velocity whose divergence results from cell proliferation, and Darcy's law to close the system. The duct membrane is described by an interface with zero thickness, across which jump conditions are imposed to link the outer and inner pressures. The use of nonlinear Kedem-Katchalsky conditions makes it possible to describe continuously the transition from DCIS to IDC. Defects on the membrane are modeled by an evolution law of its permeability, which depends on MMP concentration. Note that for the sake of simplicity, we omit the elastic tension of the membrane. The cell proliferation rate depends on the pressure and on the nutrient supply, accordingly the biological observations [9, 40]. We eventually add the influence of the chemical factors produced by the immune cells. Among them, the tumor necrosis factors (TNF) consist of a group of cytokines that may simultaneously promote the cell death but also the cancer proliferation [38, 15].

We then describe the production and the activation of MMPs enzymes. The precise pathways of MMPs production is complex and still unclear. Generally speaking, MMPs, which are produced by cancer cells, may be activated by the pro-MMPs produced by immune cells, which are located in the tumor microenvironment (TME). The effect of the TME is thus complex, and a simplified model for MMPs is presented in paragraph 2.2.

Section 3 is devoted to the numerical results. We present the growth of the tumor, the membrane degradation under the action of MMPs, and the progression of an invasive carcinoma through the membrane. Our model provides numerical results that are qualitatively in agreement with the biopsies arising from the literature. Depending on the production of MMPs, our model reproduces the different cancer phenotypes reported in biopsies (tumor confined to the duct with and without necrotic core, cancer invasion due to the duct membrane degradation). We provide in Fig. 1 the 
different phenotypes that can be described by our modeling. Interestingly, the three main patterns (DCIS, DCIS with a necrotic core and IDC after membrane degradation) are accounted for by the same theoretical framework.

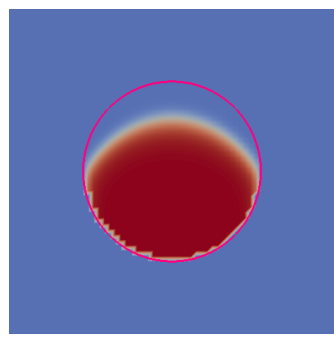

(a) DCIS without necrotic core

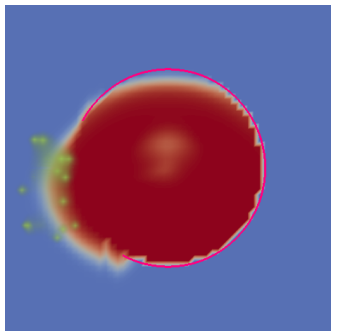

(c) Membrane degradation and cancer invasion
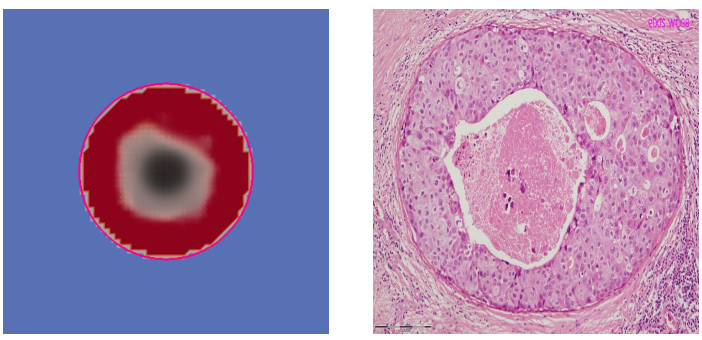

(b) DCIS with a necrotic core

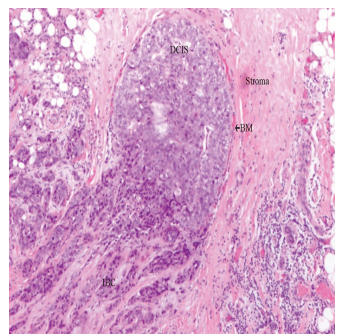

Figure 1. Main phenotypes accounted for by the model in the same theoretical framework, and comparison with biopsies. Source of biopsies: www. breastpathology.info (2013).

Remark 1 (Possible biological outcomes). Our numerical model provides a theoretical framework of the growth of ductal carcinomas, and in particular of the transition from DCIS to IDC. It makes the link between the tumor production of MMPs and the duct membrane porosity at the tumor scale. Such a modeling might be of crucial interest to test the influence of new drugs such as matrix metalloproteinase inhibitors on the cancer evolution. In particular, our numerical simulation suggests that once the membrane is degraded, MMP inhibitors seem useless since the duct membrane is already porous. On the contrary, the combination of MMP inhibitor with standard drugs might prevent the transition towards the invasive ductal carcinoma.

Moreover our numerical simulations suggest that the tumor shape provides possible information on the tumor microenvironment. In particular, in DCIS the production of TNFs by the microenvironment seems to drive the shape of the necrotic core. As pointed out before, TNFs have the paradoxical property of promoting the tumor necrosis at low concentration and the cell proliferation at higher concentration. Therefore in DCIS, the shape of the necrotic core might predict the forthcoming transition towards IDC.

\section{Tumor GROWTH MODEL OF DUCTAL CARCINOMA}

The structure of the model is outlined in Fig. 2. For the sake of clarity, we subdivide our model into two parts. The core model, presented in Subsection 2.1, describes the main mechanical 


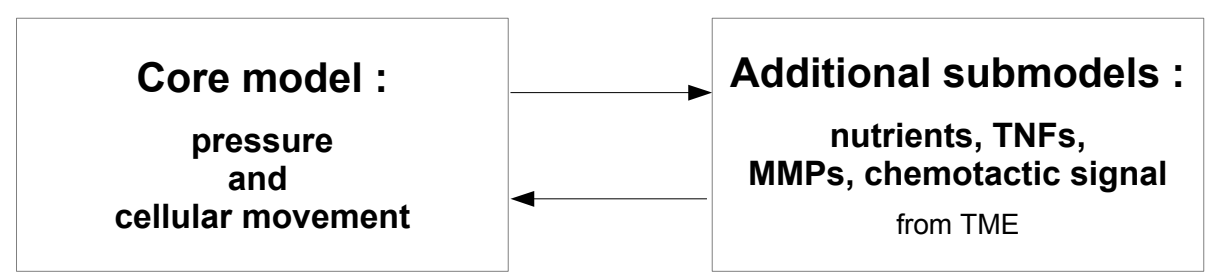

Figure 2. Schematic diagram of the model structure.

processes of the tumor growth. The model consists of a hyperbolic system of PDEs, whose main novelty lies in the transmission conditions across the membrane. Actually, most of the existing PDE models of cancer growth deal with one domain (possibly with free-boundary) however the crossing of the tumor has not been addressed, as far as we know. Specific continuity condition is imposed on the hyperbolic system to ensure the mass conservation across the membrane. Kedem-Katchalsky conditions, which are well-known in porous media modeling, are then used to describe the velocity near the interface. The second part of the model, given at Subsection 2.2, presents the additional bricks, which describe the influences of the nutrient supply, the production of MMPs and both growth and necrosis factors. The additional bricks may be changed without modifying the main core model of tumor growth, providing a test-toolbox of in silico hypotheses. It is worth noting that the model is written in a dimensionless form. We expect qualitative and phenomenological validations with biopsies. The quantitative calibration of the model on well-defined data is far beyond the scope of the paper.

2.1. Core model: cell movement and pressure equations. The domain denoted by $\Omega$ (in $2 \mathrm{D}$ or $3 \mathrm{D})$ is composed of the duct membrane $(\Gamma)$, seen as an interface, that splits $\Omega$ into two subdomains (Fig. 3): the duct $\left(\Omega_{0}\right)$, and the outer medium $\left(\Omega_{1}\right)$. The core model consists of

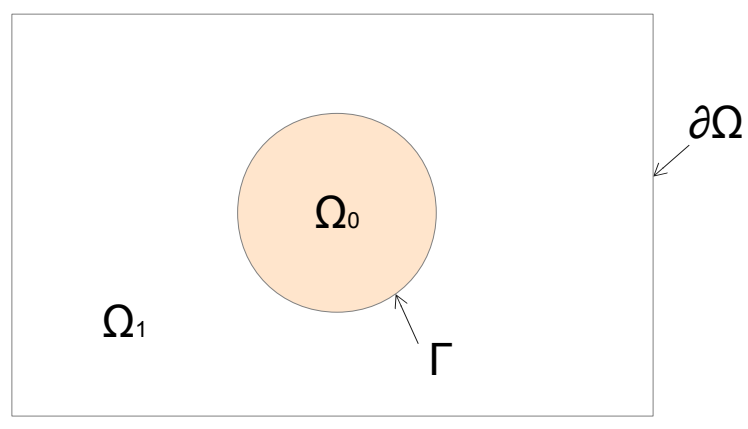

Figure 3. Geometrical framework. $\Omega$ denotes the domain of computation, $\Omega_{0}$ is the duct, $\Omega_{1}$, the stroma and $\Gamma$ denotes the duct membrane.

advection-reaction equations satisfied by the biological species densities (or proportions) ${ }^{2}$ stated piecewisely in the duct and in the surrounding tissue. More precisely, the healthy stroma cell density $(S)$ are located in the domain out of the duct, the densities of the proliferating tumor cell

\footnotetext{
${ }^{2}$ Throughout the paper, density refers to a proportion of cell and is between 0 and 1.
} 
$(P)$, and of the necrotic cell $(N)$ are originally located in the duct but they can also cross the membrane if it is degraded. Since the duct is filled up with the luminal fluid $(L)$, it is important to add its (passive) contribution on the tumor growth. All the densities are advected at the velocity $\mathbf{v}$. The transmission across the duct membrane is chosen so as to satisfy the mass conservation law.

2.1.1. Notations and hypotheses. We assume that tissues consist of the cell species that behave like an incompressible and passive fluid, driven by a homogeneous movement due to cell proliferation. Each subdomain is supposed saturated, therefore the following saturation condition on the densities holds:

$$
P+N+S+L=1, \quad \text { in } \Omega_{0} \text { and } \Omega_{1} .
$$

At the initial time, healthy cells $S$ are located out of the duct, in the surrounding stroma. The proliferative cancer cells $P$ are inside the duct, attached to the duct membrane, which is filled up with the luminal fluid $L$. The necrotic tumor cells $N$ may appear later on, due to the lack of nutrients or due to the secretion of necrosis factors by the microenvironment. The heuristics of the core model are outlined in Fig. 4.

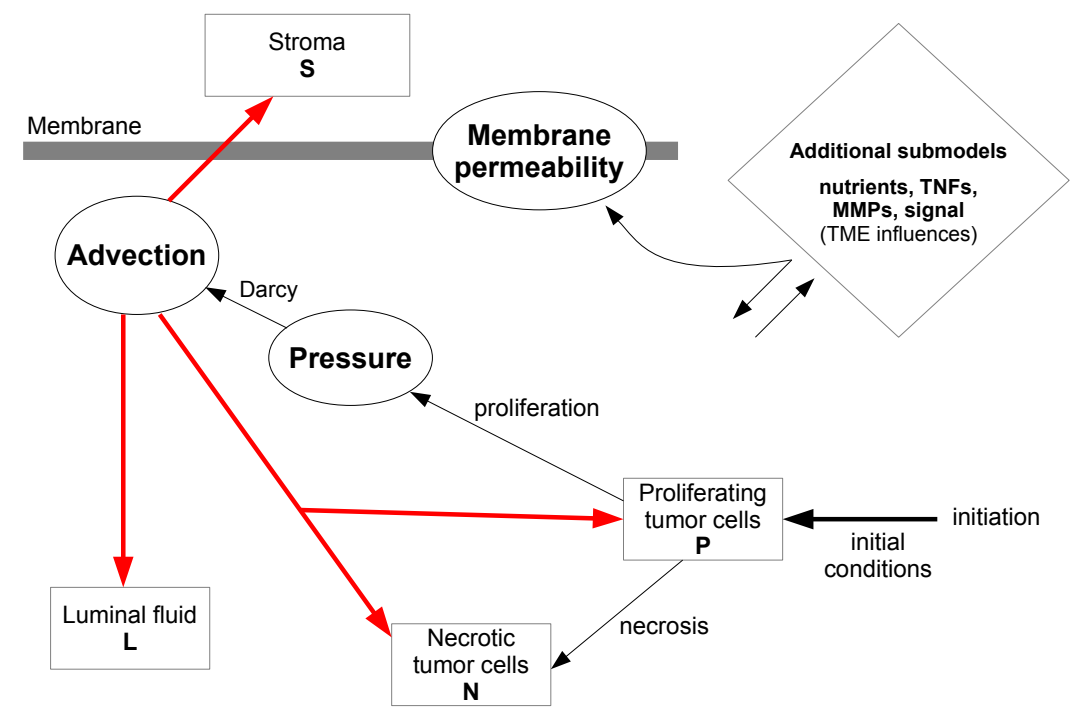

Figure 4. Schematic diagram of the core model.

After cancer initiation, tumor cells either proliferate by mitosis or turn into necrotic cells, depending on the nutrient concentration $\Theta$, the necrosis factor concentration $\tau$ and the pressure in the tumor. Proliferation generates an increase of the tumor volume, which induces the movement of all the species at the velocity v. Darcy law is imposed to close the system. The transmission of the pressure across the duct membrane is taken into account by Kedem-Katchalsky conditions. Such conditions ensure the continuity of the normal velocity across the membrane. The jump of the pressure across the membrane is proportional to the flux, the multiplicative coefficient being the membrane permeability. The membrane permeability describes the membrane degradation under the action of MMP enzymes. 
2.1.2. Advection. We assume that the cells and the luminal fluid move at the same velocity field $\mathbf{v}$, whose divergence is given by the cell proliferation. The functions $\alpha_{P}$ and $\alpha_{N}$ denote the rates of proliferation and necrosis, respectively. They will be explicitely given in subsection 2.1.4. Mass balance law implies that the general movement is governed by the following advection equations written in $\mathbb{R}^{+} \times\left(\Omega_{0} \cup \Omega_{1}\right)$ :

$$
\begin{aligned}
& \partial_{t} P+\nabla \cdot(\mathbf{v} P)=\left(\alpha_{P}-\alpha_{N}\right) P, \\
& \partial_{t} N+\nabla \cdot(\mathbf{v} N)=\alpha_{N} P, \\
& \partial_{t} S+\nabla \cdot(\mathbf{v} S)=0, \\
& \partial_{t} L+\nabla \cdot(\mathbf{v} L)=0 .
\end{aligned}
$$

Denoting by $\mathbf{X}$ the vector $(P, N, S, L)$, and by $\mathcal{A}$ the matrix

$$
\mathcal{A}=\left(\begin{array}{cccc}
\alpha_{P}-\alpha_{N} & 0 & 0 & 0 \\
\alpha_{N} & 0 & 0 & 0 \\
0 & 0 & 0 & 0 \\
0 & 0 & 0 & 0
\end{array}\right)
$$

the above system reads

$$
\partial_{t} \mathbf{X}+\nabla \cdot(\mathbf{v} \otimes \mathbf{X})=\mathcal{A} \mathbf{X}, \quad \text { in } \Omega_{0} \cup \Omega_{1} .
$$

If the basement membrane is intact, the species do not cross the interface $\Gamma$. The normal component of the cell velocity is thus zero on the interface, and species densities are discontinuous across $\Gamma$. On the contrary, if the membrane is degraded its permeability increases. The normal velocity is thus non-zero and the species can cross the porous interface. In order to satisfy the conservation law across $\Gamma$ the following transmission conditions across the membrane is imposed on $\mathbf{X}$ :

$$
\llbracket(\mathbf{v} \cdot \mathbf{n}) \mathbf{X} \rrbracket=0,
$$

where $\mathbf{n}$ is the outgoing normal vector to $\Gamma$ (from $\Omega_{0}$ to $\Omega_{1}$ ). The above brackets $\llbracket \cdot \rrbracket$ denote the jump across $\Gamma$ defined as (see Fig. 5 )

$$
\llbracket \mathbf{X} \rrbracket=\left.\mathbf{X}\right|_{\Gamma^{+}}-\left.\mathbf{X}\right|_{\Gamma^{-}} .
$$

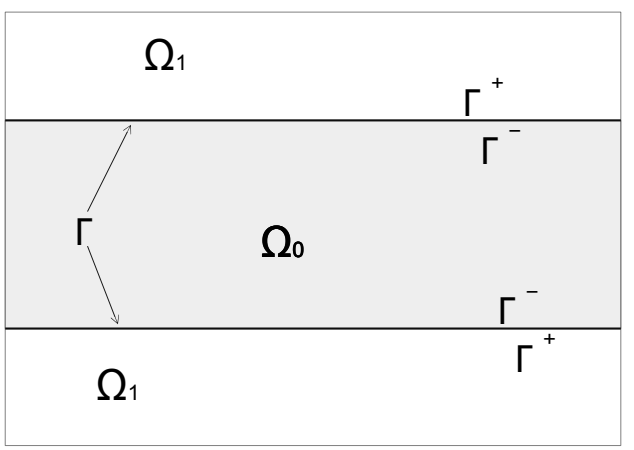

Figure 5. $\Gamma$ is considered as an interface. 
Finally, summing equations (2a) $-(2 \mathrm{~d})$ and using the saturation condition (1) lead to the divergence condition:

$$
\nabla \cdot \mathbf{v}=\alpha_{P} P .
$$

Note that unlike 24], since no treatment is accounted for, the divergence of velocity field $\mathbf{v}$ is non negative, meaning that the tumor volume increases.

Remark 2. It is worth noting that the healthy cells are not located in the duct membrane and the luminal fluid is supported only in the duct. This is imposed at the initial time. Since the divergence of the velocity is always non negative, the motion of the quantities are outwardly directed from the tumor towards its exterior. Thus healthy cells density never enters the duct and the luminal fluid hardly goes out of the duct. In order to write the system in the same framework, we extended these quantities to the whole domain by 0 .

2.1.3. Membrane permeability and Darcy law. In the simplest version of the model, the system is closed thanks to Darcy's law, as originally proposed by Greenspan [14] and later on by Ambrosi and Preziosi [1, and T. Colin, O. Saut and colleagues [3, 4, 33, 24]. For the sake of simplicity we consider that the permeability of $\Omega_{0}$ and $\Omega_{1}$ is constant and equal to $k$ and then

$$
\mathbf{v}=-k \nabla \Pi \text {. }
$$

The key point lies in the transmission conditions across $\Gamma$. Since the membrane is a thin layer with very low permeability (Fig. 5), we link the outer and inner media thanks to Kedem-Katchalsky conditions ${ }^{3}[22,29,18,25]$, which are an asymptotic version of transmission conditions across a weakly porous thin layer. More precisely, denote by $\kappa_{m}$ the surface permeability of the duct membrane. It is assumed to depend on the concentration of MMPs denoted by $M$. The following simple ordinary differential equation is used to describe the dynamics of $\kappa_{m}$ :

$$
\begin{aligned}
& \partial_{t} \kappa_{m}(t, M)=\gamma_{M} M\left(\kappa_{0}-\kappa_{m}\right), \\
& \left.\kappa_{m}\right|_{t=0}=0 .
\end{aligned}
$$

where $\kappa_{0}$ is the permeability of the deteriorated duct membrane. In other words, the membrane permeability is given by the following expression of the MMP concentration:

$$
\kappa_{m}(t, M)=\kappa_{0}\left(1-e^{-\gamma_{M} \int_{0}^{t} M(s, x) d s}\right) .
$$

The time derivative expresses the fact that the degradation of the membrane is not an instantaneous process. The pressure then satisfies the following elliptic problem:

$$
\begin{aligned}
& -\nabla \cdot(k \nabla \Pi)=\alpha_{P} P \quad \text { in } \quad \mathbb{R}^{+} \times\left(\Omega_{0} \cup \Omega_{1}\right), \\
& \llbracket k \partial_{\mathbf{n}} \Pi \rrbracket=0, \\
& \kappa_{m}(t, M) \llbracket \Pi \rrbracket=\left.k \partial_{\mathbf{n}} \Pi\right|_{\Gamma^{+}}, \\
& \left.\Pi\right|_{\partial \Omega}=0 .
\end{aligned}
$$

\footnotetext{
${ }^{3}$ Kedem-Katchalsky conditions refer to porous media, it corresponds to the so-called Kirchoff law in electromagnetic as used by C. Poignard and colleagues [29, 18, 25].
} 
The homogeneous condition on the boundary reflects the assumption that tumor growth has negligible impact on the tissue far from the tumor. The tumor pushes the healthy tissue and the luminal fluid out of the computational domain without any feedback on the internal pressure. In the above model, as soon as MMPs are produced, $\kappa_{m}$ increases. The normal component of the velocity is then strictly positive, and thanks to condition (4), the tumor cells begin to invade the surrounding tissue.

2.1.4. Proliferation and necrosis rates. The proliferation and necrosis rates are driven by chemical and mechanical environmental factors. Among them, the proliferation rate essentially depends on the nutrient concentration $\Theta$ and the pressure $\Pi$. Both have a significant impact on reducing their rate of proliferation in the case of lack of nutrients, or in the case of excessive pressure exerted on cancer cells [9, 40]. The necrosis rate also depends on the nutrient concentration $\Theta$. Moreover, both proliferation and necrosis rates may depend on environmental factors $\tau$, which can promote the cell proliferation and the cell death. For the sake of simplicity, the lack of nutrient supply is generically referred to as hypoxia.

We denote by

- $\Pi^{\max }$, the maximal pressure that cancer cells can withstand before stopping their mitoses. $\Theta_{P}$ and $\Theta_{N}$ are the hypoxia and necrosis thresholds for nutrient concentration $\Theta$. We impose

$$
0<\Theta_{N}<\Theta_{P}
$$

such that for $\Theta$ smaller than $\Theta_{N}$ cancer cells $P$ turn into necrotic cells $N$, between $\Theta_{N}$ and $\Theta_{P}$ the cells $P$ do not proliferate, but are not necrotic: they are in a quiescent phase. For $\Theta$ above $\Theta_{P}$, the cancer cells $P$ proliferate and the tumor grows,

- $\tau_{P}$ and $\tau_{N}$, the growth and necrosis thresholds for environmental factor concentration $\tau$. Beyond $\tau_{P}$ the growth factor concentration is sufficient to promote the cell proliferation, and beyond $\tau_{N}$ the necrosis factor concentration is sufficient to generate necrosis,

- $\mathcal{H}$, a regularization of the Heaviside function. For instance, for any density $Y$, we use

$$
\mathcal{H}(Y)=\frac{1+\tanh (\Lambda(Y-1))}{2} .
$$

The numerical parameter $\Lambda$ is a strictly positive non dimensional constant which drives the stiffness of the function.

The proliferation and necrosis rates are thus given by

$$
\begin{aligned}
& \alpha_{P}(\Theta, \tau, \Pi)=\bar{\alpha}_{P} \mathcal{H}\left(\Theta / \Theta_{P}\right) \mathcal{H}\left(\tau / \tau_{P}\right) \frac{\Pi^{\max }-\Pi}{\Pi^{\max }}, \\
& \alpha_{N}(\Theta, \tau)=\bar{\alpha}_{N} \mathcal{H}\left(\Theta_{N} / \Theta\right) \mathcal{H}\left(\tau / \tau_{N}\right)
\end{aligned}
$$

where $\bar{\alpha}_{P}\left(\right.$ resp. $\left.\bar{\alpha}_{N}\right)$ is the maximal proliferation (resp. necrosis ) rate.

Remark 3 (Influence of necrosis factors on both proliferation and necrosis rates). In the above definition the tumor necrosis factors concentration $(\tau)$ impacts on both $\alpha_{P}$ and $\alpha_{N}$. This is to account for the biological evidences provided by Waterson et al., and Hamai et al. [38, 15. that necrosis factors seem to promote both necrosis and proliferation of cancer cells. 
Remark 4 (Positivity of $\alpha_{P}$ ). A priori, according to equation (8a) the proliferating coefficient may be negative. However, this cannot hold thanks to the elliptic equation on $\Pi$. More precisely, the problem satisfied by $\Pi$ reads then:

$$
\begin{aligned}
& -\nabla \cdot(k \nabla \Pi)=\beta_{P} \mathcal{H}\left(\Theta / \Theta_{P}\right) \mathcal{H}\left(\tau / \tau_{P}\right) P \frac{\Pi^{\max }-\Pi}{\Pi^{\max }} \quad \text { in } \quad \mathbb{R}^{+} \times\left(\Omega_{0} \cup \Omega_{1}\right), \\
& \llbracket k \partial_{\mathbf{n}} \Pi \rrbracket=0, \\
& \kappa_{m}(t, M) \llbracket \Pi \rrbracket=\left.k \partial_{\mathbf{n}} \Pi\right|_{\Gamma^{+}}, \\
& \left.\Pi\right|_{\partial \Omega}=0 .
\end{aligned}
$$

Multiplying the negative part $4^{4}\left[\Pi^{\max }-\Pi\right]^{-}$of $\Pi^{\max }-\Pi$, and integrating by parts lead to

$$
\begin{aligned}
-\int_{\Omega} \nabla \cdot(k \nabla \Pi)\left[\Pi^{\text {max }}-\Pi\right]^{-} d x & =\int k\left|\nabla\left[\Pi^{\text {max }}-\Pi\right]^{-}\right|^{2} d x+\int_{\Gamma} \llbracket k \partial_{n} \Pi\left[\Pi^{\text {max }}-\Pi\right]^{-} \rrbracket d s, \\
& =-\int_{\Omega} \beta_{P} \mathcal{H}\left(\Theta / \Theta_{P}\right) \mathcal{H}\left(\tau / \tau_{P}\right) P \frac{\left(\left[\Pi^{\text {max }}-\Pi\right]^{-}\right)^{2}}{\Pi^{\text {max }}} \leq 0,
\end{aligned}
$$

since the term $\beta_{P} \mathcal{H}\left(\Theta / \Theta_{P}\right) \mathcal{H}\left(\tau / \tau_{P}\right) P$ is positive. Then using the continuity of the Neumann trace, and the Kedem-Katchalsky transmission condition, one can easily observe that

$$
\llbracket k \partial_{n} \Pi\left[\Pi^{\max }-\Pi\right]^{-} \rrbracket=-\kappa_{m} \llbracket \Pi^{\max }-\Pi \rrbracket \llbracket\left[\Pi^{\max }-\Pi\right]^{-} \rrbracket \geq 0,
$$

from which we infer that $\left[\Pi^{\max }-\Pi\right]^{-}=0$ and thus $\Pi \leq \Pi^{\max }$.

\subsection{Submodels for the concentrations of nutrients, MMPs and tumor necrosis factor.}

The core model involves parameters that depend on the concentrations of nutrients $\Theta$, of MMPs $M$, and of the tumor necrosis factors $\tau$. These concentrations are described below, thanks to ad hoc models designed as simple as possible in accordance with the biological knowledge. These following models can be changed without impacting on the overall structure of the above core model.

2.2.1. Nutrient supply. In the case of DCIS, nutrients are mainly provided by the vascularized duct membrane (see Fig. 6(b) ). According to Kellner et al. [19] the diffusion length of the nutrients is a few hundred microns. Moreover proliferating cells consume nutrients, therefore our simple model of nutrient concentration reads

$$
\Theta(t, \mathbf{x})=\Theta^{\max } e^{-c \delta(\mathbf{x})(1+P)}, \quad \text { with } \quad \delta(\mathbf{x})=\operatorname{dist}(\mathbf{x}, \Gamma),
$$

where $c$ is a positive constant. The distance to the vasculature can be easily estimated thanks to a level-set function and redistanciation usual techniques [36].

\footnotetext{
${ }^{4}$ We use the convention that given a real number $x$, its negative and positive part $x^{-}$and $x^{+}$are positive numbers such that

$$
x=x^{+}-x^{-}, \quad|x|=x^{+}+x^{-} .
$$
}




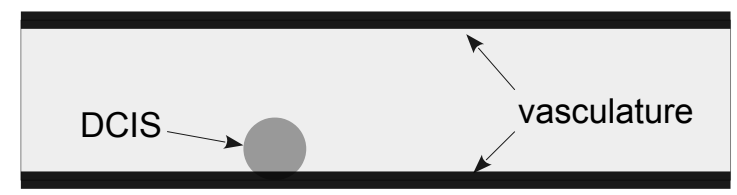

(a) Confined stage

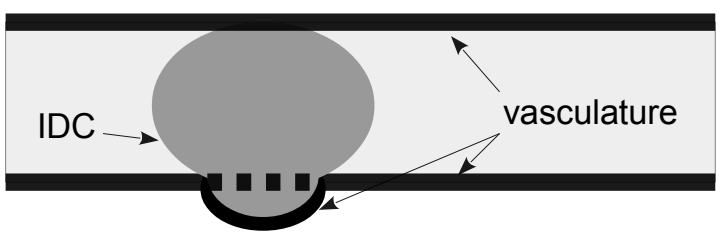

(b) Invasive stage

Figure 6. Simplistic assumptions on vasculature.

2.2.2. Tumor Necrosis Factors. TNFs are chemical substances secreted by macrophages that infiltrate the tumor. We assume macrophages are randomly distributed in the tumor [38, 15]. Let the tumor and its volume be denoted by

$$
\chi_{T}(t)=\{\mathbf{x} \in \Omega:(P+N)(t, x)>0\}, \quad\left|\chi_{T}(t)\right|=\int_{\chi_{T}(t)} d s .
$$

Denote by $d_{\tau}$ the average number of macrophages per unit volume in the tumor. The number of macrophages in the tumor is then given by the time-dependent integer function

$$
n_{\tau}(t)=\left\lceil d_{\tau}\left|\mathcal{X}_{T}(t)\right|\right\rceil
$$

Note that since the tumor keeps growing, the function $n_{\tau}$ is an increasing function of $t$. The macrophage location is given by the increasing family of random vectors $\left(\mathbf{y}_{i}\right)_{i=1, \ldots, n_{\tau}(t)}$ of $\chi_{T}(t)$ with the same uniform probability distribution. The probability density function is thus given by

$$
\forall(t, \mathbf{x}), f_{\tau}(t, \mathbf{x})=\frac{1}{\left|\chi_{T}(t)\right|} \mathbb{1}_{\chi_{T}(t)}(\mathbf{x}) .
$$

Denoting by $\gamma_{\tau}$ the production rate of macrophages, the spatial distribution of TNF concentration is given by

$$
\begin{aligned}
-\Delta \tau=0 & \text { in } \Omega \backslash \omega_{\tau}, \\
\left.\tau\right|_{\partial \Omega}=0, & \left.\tau\right|_{\omega_{\tau}}=\gamma_{\tau} .
\end{aligned}
$$

2.2.3. MMPs enzymes: the keypoint of membrane degradation. The duct membrane confines the tumor and prevents the cancer invasion in the surrounding tissue. However some mutated cancer cells can produce specific enzymes that degrade the membrane. Once the membrane is degraded, tumor is no more confined to the duct and cell divisions restart. The tumor cells cross the membrane and then invade the surrounding tissue (Fig.7). The action of MMPs enzymes is a complex question, which has been extensively studied by the biological community but it still remains unclear [7, 28, 23. According to the literature, different kinds of MMPs seem to be involved: diffusive MMPs and MT1-MMPs, which are embedded in the cytoplasmic membrane and do not diffuse. MT1-MMPs are crucial in cell migration. They provide to cell the ability to degrade collagen fibers of the extracellular matrix. However, these MT1-MMPs have to be activated. It is established that in response to a chemical signal of the tumor, fibroblasts of the immune system migrate through the stroma towards the duct, and produce inactive proenzymes. These pro-enzymes called pro-MMPs 


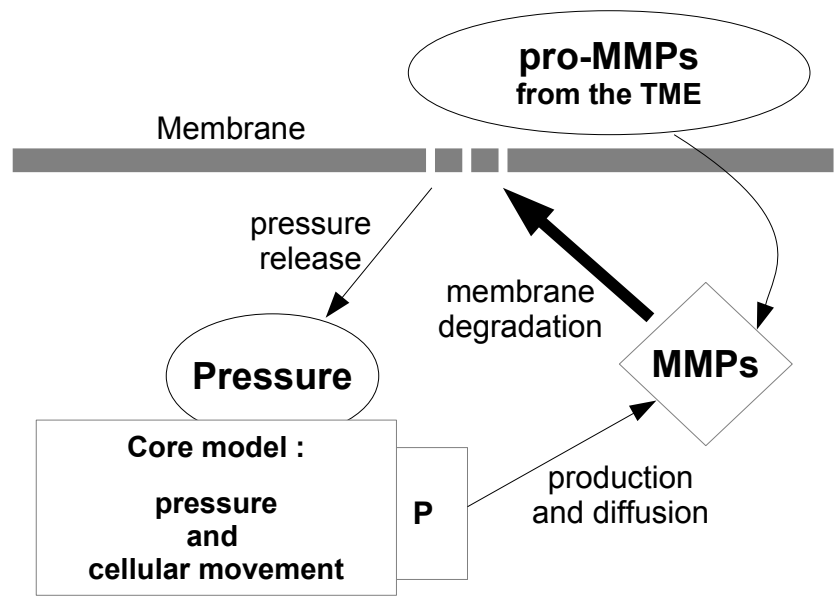

Figure 7. Schematic diagram of MMP model.

are insidious. Indeed, when combined to MT1-MMPs, they form chemical complexes, which activate the MT1-MMP and lead to membrane proteolysis [5, 6, 17, 32,

To describe this complex phenomenon, we denote respectively by $M^{\star}$ and $M^{T}$ the pro-MMPs produced by the fibroblast and the MT1-MMPs acquired by mutated cancer cells. By analogy with macrophages, we introduce the number of fibroblasts per unit volume, $d_{M^{\star}}$, and the time dependent number $n_{M^{\star}(t)}$ of pro-MMPs in the stroma, so that

$$
n_{M^{\star}(t)}=\left\lceil d_{M^{\star}}\left|\chi_{S}(t)\right|\right\rceil, \quad \chi_{S}(t)=1-\chi_{T}(t) .
$$

Then, the fibroblasts are the random vectors $\left(\mathbf{y}_{i}\right)_{i=1, \ldots, n_{M^{\star}}}$ whose probability density function is given by

$$
\forall(t, \mathbf{x}), f_{\star}(t, \mathbf{x})=\frac{1}{\left|\chi_{S}(t)\right|} \mathbb{1}_{\chi_{S}(t)}(\mathbf{x}) .
$$

Pro-MMPs are then supposed to diffuse from the fibroblasts. The concentration $M^{\star}$ thus satisfies

$$
\begin{aligned}
-\Delta M^{\star}=0 & \text { in } \Omega \backslash \omega_{\star}, \\
\left.M^{\star}\right|_{\partial \Omega} & =0,\left.\quad M^{\star}\right|_{\omega_{\star}}=\gamma_{\star} .
\end{aligned}
$$

The presence of MT1-MMPs, of concentration $M^{T}$, depends on whether or not the cancer cells have acquired the genetic ability to express them. The increase of pressure blocks the cell proliferation in the tumor, but it also promotes genetic mutations that lead to EMT for cancer cells that still proliferate [40, 37]. In particular, the probability of the emergence of MT1-MMPs increases when the tumor turns into quiescent phase (that is when $\alpha_{P}$ decreases). We thus assume the following ordinary differential equation satisfied by $M^{T}$ :

$$
\partial_{t} M^{T}(t, x)=\mathcal{H}\left(\Pi / \Pi_{t h}\right) P(t, x) \bar{M}_{0}^{T},
$$

where $\bar{M}_{0}^{T}$ is the maximal rate of production of MT1-MMP by mutated cancer cells. 
We then define the concentration $M$ in complexes proMMP/MT1-MMP by

$$
M(t, x)=\mathcal{H}\left(M^{\star} / M_{t h}\right) M^{T}(t, x) .
$$

2.3. The complete model. Let us summarize the complete model. Let $\mathbf{X}=(P, N, S, L)$. Denote by $a=\left(\alpha_{P}, 0,0,0\right)$ and let $\mathcal{A}$ be a matrix in $\mathcal{M}_{4}\left(\mathbb{R}^{+}\right)$such that

$$
\mathcal{A}_{1,1}=\alpha_{P}-\alpha_{N}, \quad \mathcal{A}_{2,1}=\alpha_{N}, \quad \mathcal{A}_{i, j}=0, \forall(i, j) \notin\{(1,1),(2,1)\} .
$$

Then, for any $t \in \mathbb{R}^{+}$, the complete model is given by

Core model

- advection equations in $\Omega_{0} \cup \Omega_{1}$

- membrane permeability on $\Gamma$ $\partial_{t} \kappa_{m}(t, M)=\gamma_{M} M\left(\kappa_{0}-\kappa_{m}\right)$.

Nutrients, TNF and MMPs submodels

- nutrients in $\Omega_{0} \cup \Omega_{1}$

$$
\Theta(t, \mathbf{x})=\Theta^{\max } \mathrm{e}^{-c \delta(\mathbf{x})(1+P)}, \quad \text { with } \quad \delta(\mathbf{x})=\operatorname{dist}(\mathbf{x}, \Gamma),
$$

- TNF equation in $\Omega$

$$
-\Delta \tau=0 \quad \text { in } \Omega \backslash \omega_{\tau},\left.\quad \tau\right|_{\partial \Omega}=0,\left.\quad \tau\right|_{\omega_{\tau}}=\gamma_{\tau} .
$$

- MMP equations in $\Omega$

$$
\begin{aligned}
& -\Delta M^{\star}=0 \quad \text { in } \Omega \backslash \omega_{\star},\left.\quad M^{\star}\right|_{\partial \Omega}=0,\left.\quad M^{\star}\right|_{\omega_{\star}}=\gamma_{\star}, \\
& \partial_{t} M^{T}(t, x)=\mathcal{H}\left(\Pi / \Pi_{t h}\right) P(t, x) \bar{M}_{0}^{T} \\
& M=\mathcal{H}\left(M^{\star} / M_{t h}\right) M^{T} .
\end{aligned}
$$




\section{NumericAl RESUlts}

This section is devoted to investigate numerically the different behaviors reproduced by the model. We first present briefly the numerical methods and the geometrical framework of the simulations. Then DCIS tumor growth are presented and we conclude by showing how the production of MMPs makes it possible to account for the transition from the in situ to the invasive phases.

3.1. Numerical methods. The membrane $\Gamma$ is given as the zero of a level-set function $\psi$, and the domain $\Omega$ is mesh in by a Cartesian grid. $\Gamma^{h}$ denotes the intersection of the grid and the level-set, and $\Omega^{h}$ denotes the set of the grid points.

We first present the geometrical framework used for the computations. The numerical method is then presented. The difficulty lies in the method used to tackle the ellipic problem satisfied by the pressure (11d)-11e. The numerical method is inspired from recent works of O. Gallinato and C. Poignard [12, 13, in which the elliptic operator and the Neumann boundary condition are discretized thanks to a stabilized version of the usual Ghost fluid method [8], based on the continuity of the stencils. Standard upwind schemes are considered to compute the advectionreaction equations, and forward Euler time-schemes are used for the time discretization. It is worth noting that our numerical method is sharp in the sense that no artificial widening of the membrane is needed. The fluxes are discretized precisely at the interface, providing sharp and accurate description of the nonlinearities that hold at the interface.

3.1.1. Geometrical framework. The simulations are computed in the domain $\Omega=[0,1]^{3}$, on a $80 \times 80 \times 40$ mesh. For the sake of simplicity, the duct is a cylinder (Fig. 8) of diameter $r_{d}$. Moreover, the cylinder axis is in the direction $(O z)$, which considerably reduces the computation time since the jump conditions $(11 \mathrm{~b})$ and $(11 \mathrm{e})$ on the interface $\Gamma$ can be treated only in the bidimensional plan $O x y$. The value of the parameters, if not specified, are given in Table 1 in

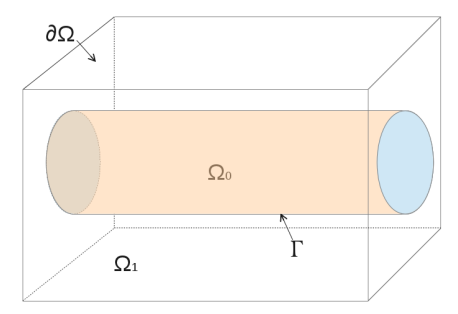

Figure 8. Domain $\Omega$ for simulations.

Appendix. For each simulation, we assume that a small tumor, supported by the membrane inside the duct, has been already initiated at the initial time. It is composed of tumor cells and defined by a smooth exponential function:

$$
P^{0}=0.1 \frac{\mathrm{e}^{-500\left|\mathbf{x}-\mathbf{x}_{0}\right|}}{\max _{\mathbf{x} \in \Omega_{0}} \mathrm{e}^{-500\left|\mathbf{x}-\mathbf{x}_{0}\right|}} \mathcal{X}_{\Omega_{0}},
$$


where $\mathbf{x}_{0}=\left(0,-r_{d}, 0\right)$ and $\mathcal{X}_{\Omega_{0}}$ is the characteristic function of $\Omega_{0}$. Then, the vector $X$ of species is initialized with

$$
X^{0}=\left.(P, N, S, L)\right|_{t=0}=\left\{\begin{array}{l}
\left(P^{0}, 0,0,1-P^{0}\right) \quad \text { in } \Omega_{0} \\
(0,0,1,0) \text { in } \Omega_{1} .
\end{array}\right.
$$

3.1.2. Laplace operator discretization. The Laplace operator is discretized direction by direction. For the sake of clarity only the $x$-direction is presented here. The quantity $u^{h}$ generically denotes the numerical approximation of $\Pi$. At the regular points are the grid points located far from the interface -all the 6 closest neighbours belong to the same subdomain $\Omega_{0}$ or $\Omega_{1}$ - the usual centered stencil is used, that is

$$
\partial_{x x}^{h} u_{i}^{h}=\frac{u_{i+1}^{h}-2 u_{i}^{h}+u_{i-1}^{h}}{h^{2}}, \quad \text { at the regular points. }
$$

Near the interface the centered discretization is not possible since one (at least) of the neighbors is on the other side of the interface. At this point, the value is called ghost value and is linearly extrapolated. For instance, the scheme for the second $x$-derivative in Fig.9 is given by

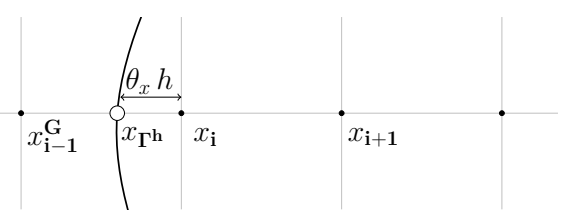

Figure 9. Ghost-Fluid method for the points near the interface.

$$
\partial_{x x}^{h} u_{i}^{h}=\frac{2}{\theta_{x}\left(1+\theta_{x}\right) h^{2}} u_{\Gamma^{h}}-\frac{2}{\theta_{x} h^{2}} u_{i}^{h}+\frac{2}{\left(1+\theta_{x}\right) h^{2}} u_{i+1}^{h}, \quad \text { near } \Gamma^{h}
$$

where $\theta_{x} h$ denotes the distance of the point $x_{i}$ to the numerical interface $\Gamma^{h}$ in the $x$-axis direction. If the case $\theta_{x}=1$ occurs, we have $u_{\Gamma^{h}}=u_{i-1}^{h}$. The scheme (14) is then equivalent to the standard 3 -point stencil scheme 13 . Conversely, if $\theta_{x}=0$, the point $x_{i}$ is an interface point and does not belong to the considered domain. It is worth noting that linear extrapolations give a nonconsistent operator near the interface while it is second order accurate at the regular points.

3.1.3. Flux discretization. The normal components $n_{x}^{\Gamma^{h}}$ and $n_{y}^{\Gamma^{h}}$ are interpolated at the order 2 as the gradient of the level-set $\psi$. The flux condition at the interface is discretized by evaluating the derivatives of $u^{h}$ on $\Gamma^{h}$. We therefore introduce new operators $\partial_{x}^{\Gamma^{h}}$ and $\partial_{y}^{\Gamma^{h}}$, that are directly or indirectly computed from other grid points, depending on whether the interface point belongs to the $x$-axis or to the $y$-axis. In Fig. 10 , the point $A$ is on the $y$-axis and the indirect $x$-derivative $\partial_{x}^{\Gamma^{h}} u_{\Gamma^{h}}^{A}$ is computed at the order 1 from the values $u_{\Gamma^{h}}^{A}$ and $u_{1}^{A}$, still using the ghost fluid method and the linear extrapolation:

$$
\partial_{x}^{\Gamma^{h}} u_{\Gamma^{h}}^{A}=\frac{u_{1}^{A}-u^{s}}{2 h}=\frac{u_{1}^{A}-u_{\Gamma^{h}}^{A}}{h}
$$




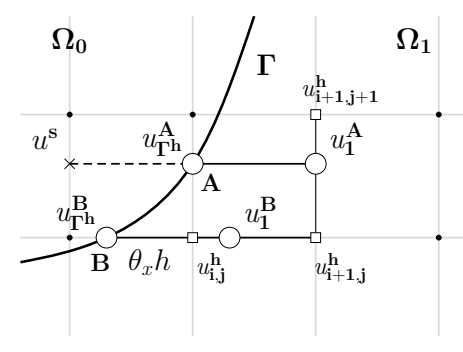

Figure 10. Stencil of the interface $x$-derivative, as presented in Gallinato and Poignard [12, 13].

where $u^{s}$ is a ghost value, which is extrapolated at the order 2 from $u_{\Gamma^{h}}^{A}$ and $u_{1}^{A}$. The intermediate value $u_{1}^{A}$ is then interpolated at the order 2 from the known values $u_{i+1, j}^{h}$ and $u_{i+1, j+1}^{h}$. The direct $x$-derivative $\partial_{x}^{\Gamma^{h}} u_{\Gamma^{h}}^{B}$ is computed in the same way:

$$
\partial_{x}^{\Gamma^{h}} u_{\Gamma^{h}}^{B}=\frac{u_{1}^{B}-u_{\Gamma^{h}}^{B}}{h},
$$

where the intermediate value $u_{1}^{B}$ is interpolated from the known values $u_{i, j}^{h}$ and $u_{i+1, j}^{h}$, which gives

$$
\partial_{x}^{\Gamma^{h}} u_{\Gamma^{h}}^{B}=\frac{-u_{\Gamma^{h}}^{B}+\theta_{x} u_{i, j}^{h}+\left(1-\theta_{x}\right) u_{i+1, j}^{h}}{h} .
$$

An example of complete stencil is shown in Fig. 11.

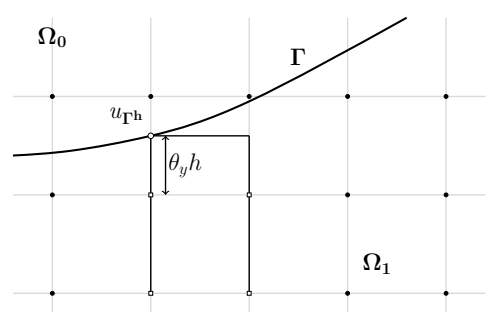

Figure 11. Complete stencil for the discretization of the Neumann boundary condition.

3.1.4. Discretization of the advection-reaction equations. Once the pressure is computed, the velocity field is obtained by discretizing the gradient of $\Pi$. First order upwind scheme is used for the space derivative. The time-scheme is the forward Euler scheme.

3.2. Early phase of DCIS growth: the influence of nutrients and TNF. We first consider a carcinoma in situ, without any production of MMPs, by setting $\bar{M}_{0}^{T}=0$. The tumor remains thus confined to the duct. We aim at investigating the influence of nutrients and TNF on the emergence of a necrotic core in the tumor.

3.2.1. Simulation of a simple DCIS growth (simulation 1). First, TNFs are not taken into account $\left(\gamma_{\tau}=0\right)$, and we assume that there is no lack of nutrient inside the tumor $(c=0)$. The tumor fills up the duct (solid carcinoma). This is shown by the longitudinal and axial cross sections in Fig. 13 and in the corresponding $3 \mathrm{D}$ simulation 12 . 


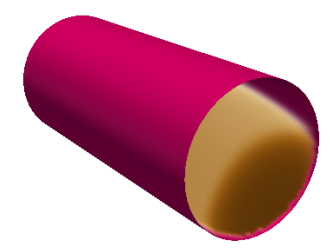

Figure 12. 3D ductal carcinoma in situ corresponding to the longitudinal and axial sections of Fig $13, t=0.5$.

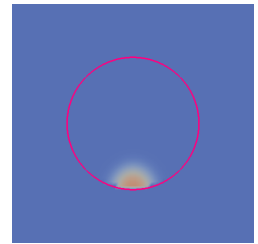

(a) $t=0.1$

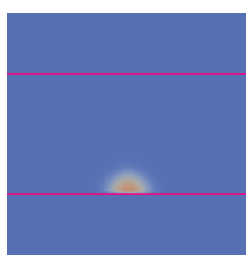

(e) $t=0.1$

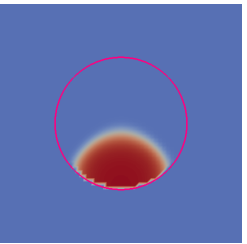

(b) $t=0.3$

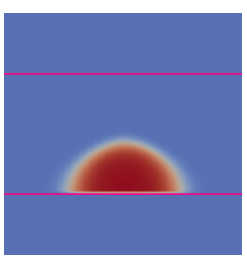

(f) $t=0.3$

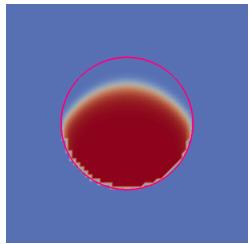

(c) $t=0.5$

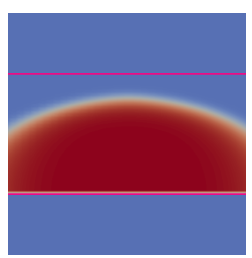

(g) $t=0.5$

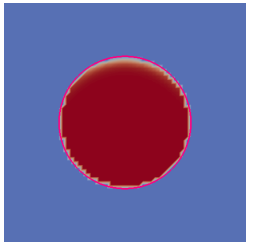

(d) $t=0.7$

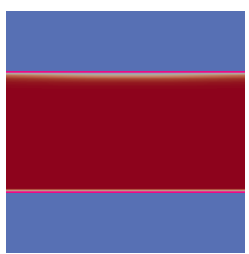

(h) $t=0.7$

Figure 13. Evolution of ductal carcinoma in situ (DCIS) in cross and longitudinal section. The tumor grows and fills up the duct (simulation 1). Time points are on an arbitrary timescale.

Simulations confirm that the model describes correctly the growth in situ, thanks to the jump conditions on the pressure. Since no MMPs are produced, homogeneous Neumann condition is imposed to the pressure and thus the normal velocity is null. The tumor remains confined to the duct. It is worth noting that the tumor grows faster in the direction of the duct axis, as observed in longitudinal section, due to the homogeneous Dirichlet condition imposed to the pressure on the boundary. This artifact can be fixed by imposing a non-homogeneous condition, possibly timedependent and proportional to the tumor volume.

3.2.2. Influence of nutrients and necrotic factors (simulations 2 and 3). Figure 14 is obtained by adding the radial decay of the nutrient $(c=2$ in equation (9) (simulation 2a). The radial distribution of the nutrients is given by (Fig. 14(a). If the duct radius is sufficiently large, the proliferating area of the tumor is ring-shaped (Fig. 14(b)). Towards the center, the tumor cells are quiescent and some of them may become necrotic (Fig. 14(c)].

Figure 15, obtained by modulating the source $\Theta^{\max }$ (simulation 2b), shows a larger quiescent 


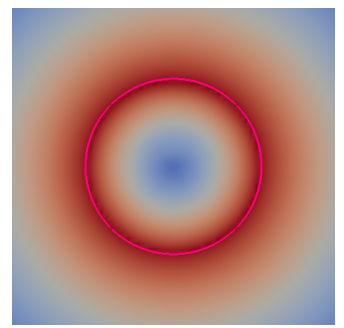

(a) nutrients $\Theta$

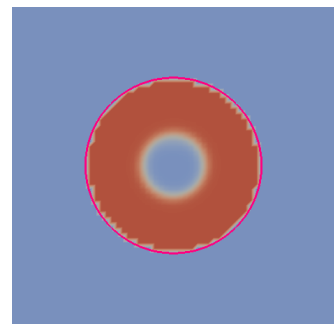

(b) proliferation area

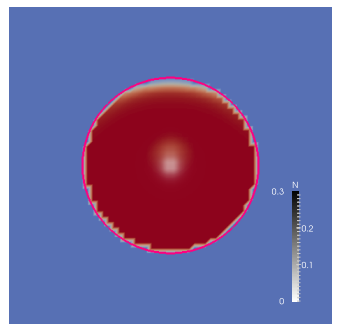

(c) tumor

Figure 14. Effect of nutrient concentration on proliferation: $\Theta^{\max }=1, t=0.8$ (simulation $2 \mathrm{a}$ ).

area. The tumor is no longer proliferating, when the nutrient concentration is below the hypoxia threshold. For $\Theta$ below the threshold of necrosis, cell death occurs, and a necrotic core appears (Fig. 15(b)-Fig. 15(c)]. This kind of confined tumor is the very early stage of breast cancer often referred to as comedocarcinoma.

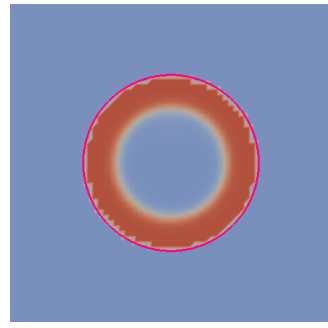

(a) proliferation area

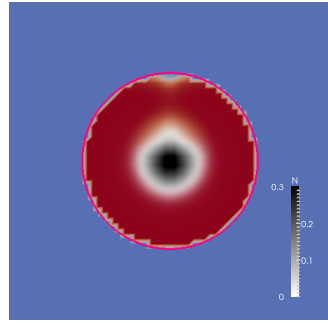

(b) tumor with a necrotic core

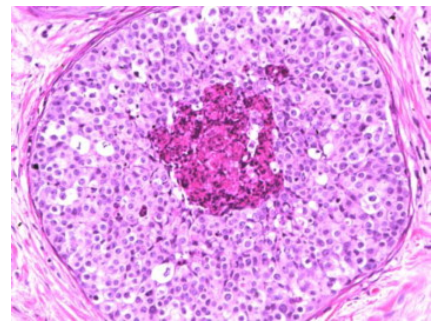

(c) biopsy of DCIS referred to as a comedocarcinoma breast cancer 34.

Figure 15. Effect of nutrient concentration on proliferation: $\Theta^{\max }=0.8, t=1$ (simulation $2 \mathrm{~b}$ ).

For the same given concentration of nutrients, the rate of necrosis increases if TNFs are introduced $\left(\gamma_{\tau}=1\right)$, with a concentration that exceeds the threshold $\tau_{N}$. Simulation 3 in Figure 16 reveals a more extensive and irregular necrosis than if only hypoxia is involved (Fig. 15). The wider necrosis is due to the increase in necrosis rate, and its irregularity to the random distribution of infiltrating macrophages. These numerical experiments tend to confirm that in addition to hypoxia due to a lack of nutrient supply, the necrotic core observed in DCIS may be explained by the action of TNF. In particular, these simulations suggest that the shape of the necrotic core may be correlated to the proportion of TNF.

3.3. Impact of MMPs on the transition from the in situ to the invasive phenotypes. In this section, we investigate the impact of the production of MMPs on the tumor invasion of the surrounding tissue. We keep the same parameters as in simulation $2 \mathrm{a}\left(c=2, \Theta^{\max }=1\right.$ and $\left.\gamma_{\tau}=0\right)$ for nutrients and TNFs. Setting $\gamma_{T}=\gamma_{\star}=1$ and $d_{M^{T}}=0.002, d_{M^{\star}}=0.01$, MMPs are produced and activated from randomly located sources. The result of simulation 5 is given in Fig. 17, showing two invasion spots. 


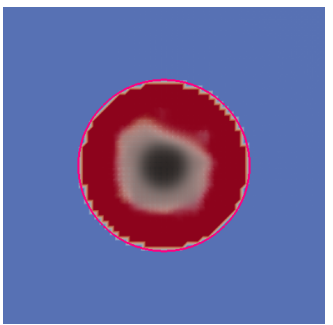

(a) necrotic area

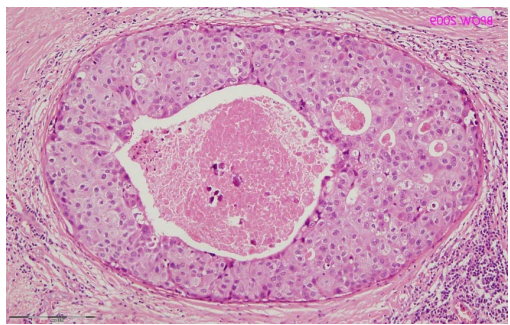

(b) biopsy of DCIS referred to as a comedocarcinoma breast cancer. Source: www . breastpathology . info (2013)

Figure 16. Effect of TNF concentration on necrosis at $t=0.8$ (simulation 3 ).

As the MMP diffusion is short-range (Fig. 17(a) , the gaps in the membrane are very localized and their number is determined by the very low number $d_{M^{T}}=0.002$ of MMP sources by unit volume of tumor cells.

Assuming that the fibroblasts are randomly located just in a given area of the stroma, and imposing a higher number per unit volume of MT1-MMP sources $\left(d_{M^{T}}=0.01\right)$, we obtain Fig. 18 (simulation 5). The location of fibroblasts implies a privileged area of invasion at the early stage. Note that since the nutrient supply is assumed to come from the duct membrane, the tumor grows also along the duct in a second step, as provided by Fig, 18(b).

\section{Conclusion And Perspectives}

We derived a continuous model, based on PDEs, which makes it possible to simulate the transition from a DCIS to an IDC and to observe the occlusion of a milk duct by a carcinoma and the early stage of invasion. From the modeling point of view, the main novelty lies in the coupling of usual advection-reaction equations with a specific elliptic problem on the pressure. More precisely, the pressure is piecewise harmonic -in the duct membrane and outside- and Kedem-Katchalsky jump conditions links the pressure of the two domains. These conditions account for the membrane permeability.

Phenomenological law (6) links the production of MMPs and the membrane permeability. Thanks to the Kedem-Katchalsky conditions, we thus describe the transition from the in situ stage to the invasive cancer due the membrane degradation by MMPs. Such a macroscopic model could be a crucial tool to test in silico the influence of new drugs such as MMP inhibitors. Moreover, as an interesting numerical output of the model, one can notice that the shape of the necrotic core seems to provide specific information on the influence of the microenvironments and the presence of TNFs. Actually, according to simulation $16(\mathrm{a})$ the production of TNFs on the microenvironment affects the shape of the necrotic core. This has to be confirmed in forthcoming studies but this could have a dramatic impact on the prediction of the transition time from DCIS to IDC.

As forthcoming works, one can point out specific patterns of breast cancer that are not accounted for by our modeling. These patterns arise in different types of DCIS: micropapillary, papillary or 


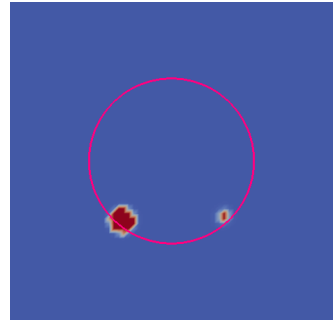

(a) MMP concentration

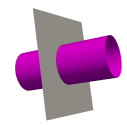

(c) Cross section

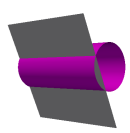

(h) Longitudinal section

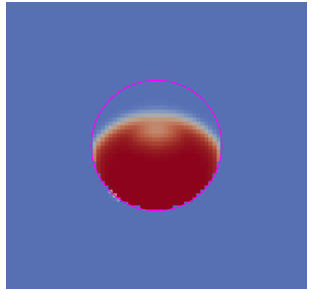

(d) $t=0.48$

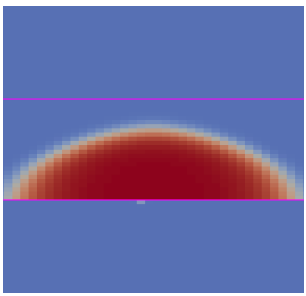

(i) $t=0.48$

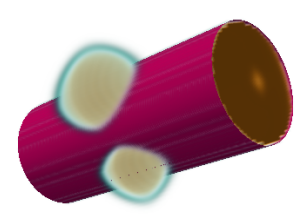

(b) Invasion in $3 \mathrm{D}(t=0.85)$

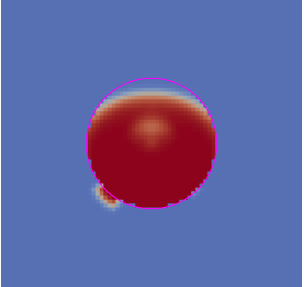

(e) $t=0.63$

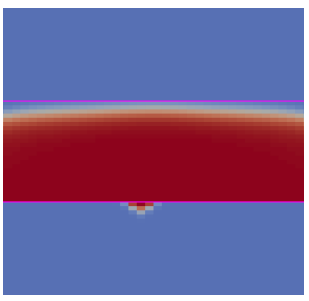

(j) $t=0.63$

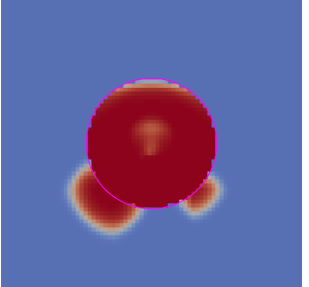

(f) $t=0.80$

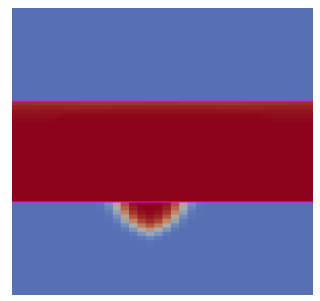

(k) $t=0.80$

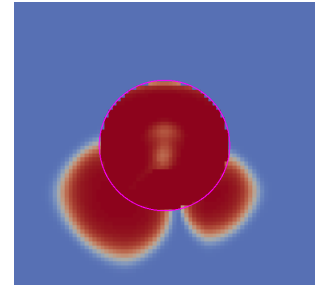

(g) $t=0.90$

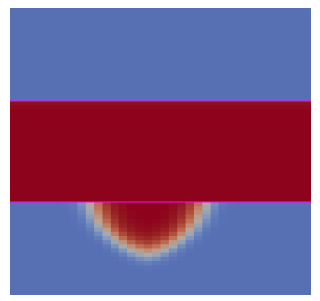

(1) $t=0.90$

Figure 17. Evolution of invasive ductal carcinoma with a variable concentration of MMPs, in cross and longitudinal section (simulation 4). Time points are on an arbitrary timescale.

cribriform carcinomas (Fig. 19). Probably this is due to the use of Darcy law, which describes the cell movement at the macroscopic scale regardless of the cell polarity and adhesions. These subcellular phenomena lead to specific directions of growth. Discrete models [27, 21, 20] as proposed by [27, 21, 20] are well appropriate to tackle these phenomena and forthcoming works could consist in deriving a new macroscopic closure law that accounts for these subcellular phenomena.

Our macroscopic and continuous approach presents the great advantage to consider cell densities, which makes it possible to simulate larger tumors, in a three-dimensional framework. Above all, our model involves much fewer parameters than discrete models. This is a crucial advantage for calibration purposes and also in order to test many biological hypotheses. Moreover, our numerical results are stable in the sense that they do not depend on the size of the mesh, unlike discrete model for which the number of cells depends of the refinement of the mesh. 


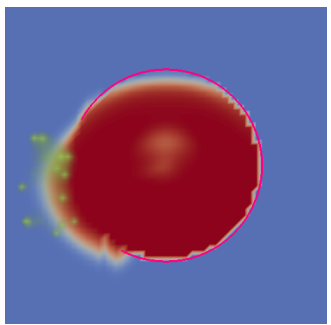

(a) Invasion and localization of the proMMP sources $(t=$ $0.75)$

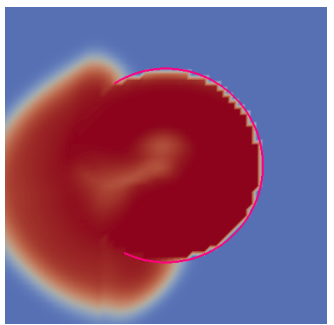

(b) Invasion $(t=0.9)$

Figure 18. IDC with restricted localization of immune cells, which produce pro-MMPs (simulation 5).

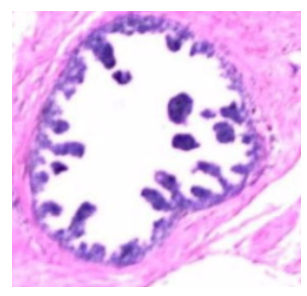

(a) micropapillary

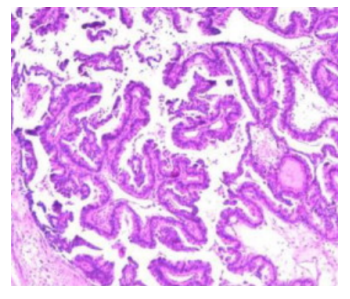

(b) papillary

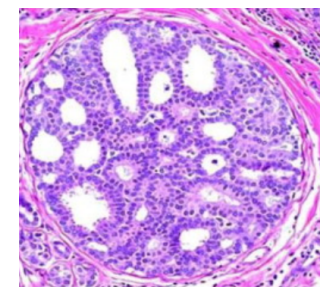

(c) cribriform

Figure 19. Specific patterns of other types of intraductal carcinoma that cannot be accounted for by the model, from 34.

In this preliminary study, the MMPs production and action have been specifically investigated, since the link between MMPs, duct membrane permeability and invasion is one of the cornerstones in the transition from DCIS to IDC. Influences of the microenvironment on the membrane degradation have also been tested. Simulations highlighted the degradation of the membrane and the spread of malignant cells outside the duct, leading to premetastatic cell migration and tumor invasion. Interestingly our numerical results support the idea that microenvironmental factors play a crucial role in tumor progression, as we are able to reproduce biopsy observations. This point is in agreement with many studies in oncology [2, 6, 16].

Hence, the qualitative behavior of our model for invasion leads to the same kind of conclusions as for the advanced hybrid model recently presented by Kim et al. in [21, 20]. Our continuous description involves less parameters, and it allows 3D simulations. Moreover, the core model is very flexible and easy to enrich with many additional submodels, in order to test other hypotheses. For instance, we could further study the anisotropy of the neighboring stroma, the action of MMPs on its ECM or the involving of different kinds of MMPs in proteolysis. A more deterministic movement of fibroblasts and macrophages could also bring more predictability to the model. The calibration of the parameters requires specific biological and medical data. This will be the next step of this study. 


\section{ACKNowledgements}

This study have been partly granted by the Plan Cancer projects MIMMOSA (Inserm 8695), DYNAMO (Inserm 9749) and NUMEP (Inserm 11099). This study has been carried out within the scope of the Inria Associate Team Num4SEP.

The numerical experiments presented in this paper were carried out using the PLAFRIM experimental testbed, being developed under the Inria PlaFRIM development action with support from LABRI and IMB and other entities: Conseil Régional d'Aquitaine, FeDER, Université de Bordeaux and CNRS (see https://plafrim.bordeaux.inria.fr/).

\section{REFERENCES}

[1] D. Ambrosi and L. Preziosi. On the closure of mass balance models for tumor growth. Mathematical Models and Methods in Applied Sciences., Vol. 12, No. 5, pages 737-754, 2002.

[2] M. Bissell and W. Hines. Why don't we get more cancer? A proposed role of the microenvironment in restrainning cancer progression. Nat Med., 17, pages 320-329, 2011.

[3] D. Bresch, T. Colin, E. Grenier, B. Ribba, and O. Saut. A viscoelastic model for avascular tumor growth. Discrete and continuous dynamical systems, supplement 2009, pages 101-108, 2009.

[4] D. Bresch, T. Colin, E. Grenier, B. Ribba, and O. Saut. Computational modeling of solid tumor growth : the avascular stage. SIAM Journal on Scientific Computing, 32(4), pages 2321-2344, 2010.

[5] O. Brummer, S. Athar, L. Riethdorf, T. Löning, and H. Herbst. Matrix-metallo-proteinases 1, 2 and 3 and their tissue inhibitors 1 and 2 in benign and malignant breast lesions : an in situ hybridization study. Virchows Arch., 435, pages 566-573, 1999.

[6] M. Cichon, A. Degnim, D. Visscher, and D. Radisky. Microenvironmental influences that drive progression from benign breast disease to invasive breast cancer. J Mammary Gland Biol Neoplasia, 15, pages 389-397, 2010.

[7] M. Duffy, T. Maguire, A. Hill, and E. McDermott. Metalloproteinases: role in breast carcinogenesis, invasion and metastasis. Breast Cancer Res., 2, pages 252-257, 2000.

[8] R. P. Fedkiw, T. Aslam, B. Merriman, and S. Osher. A Non-oscillatory Eulerian Approach to Interfaces in Multimaterial Flows (the Ghost Fluid Method). Journal of Computational Physics, 152(2):457-492, 1999.

[9] A. S. for Cell Biology. Externally applied forces can phenotypically revert malignant breast epithelial structures. In ASCB, 2012 annual meeting abstracts, pages 983, No.1673, Dec 17, 2012. http://www.ascb.org/files/ Past-AM-Meetings/2012_Abstracts.pdf.

[10] S. Franks, H. Byrne, J. King, J. Underwood, and C. Lewis. Modelling the early growth of ductal carcinoma in situ of the breast. J Math Biol., 47:424-452, 2003.

[11] S. Franks, H. Byrne, J. Mudhar, J. Underwood, and C. Lewis. Mathematical modelling of comedo ductal carcinoma in situ of the breast. Math. Med. Biol., 20:277-308, 2003.

[12] O. Gallinato, M. Ohta, C. Poignard, and T. Suzuki. Free boundary problem for cell protrusion formations: theoretical and numerical aspects. 2016.

[13] O. Gallinato and C. Poignard. Superconvergent second order Cartesian method for solving free boundary problem for invadopodia formation. 2016.

[14] H. Greenspan. On the growth and stability of cell cultures and solid tumors. Journal of Theoretical Biology, 56(1):229 - 242, 1976.

[15] A. Hamaï, J. Muret, A. Cavalcanti, S. Bonvalot, and S. Chouaïb. Le facteur de nécrose tumorale : de la biologie à la thérapie oncologique. Hématologie, 15(4), pages 291-304, 2009.

[16] M. Hu and K. Polyak. Microenvironmental regulation of cancer development. Curr Opin Genet Dev., 18(1), pages 27-34, 2008. 
[17] M. Hu, J. Yao, D. Carroll, S. Weremowicz, H. Chen, D. Carrasco, A. Richardson, S. Violette, T. Nikolskaya, Y. Nikolsky, E. Bauerlein, W. Hahn, R. Gelman, C. Allred, M. Bissel, S. Schnitt, and K. Polyak. Regulation of in situ to invasive breast carcinoma transition. Cancer Cell, 13, pages 394-406, 2008.

[18] O. Kavian, M. Leguèbe, C. Poignard, and L. Weynans. "Classical" Electropermeabilization Modeling at the Cell Scale. J. Math. Biol., 68(1-2):235-265, 2014.

[19] K. Kellner, G. Liebsch, I. Klimant, O. S. Wolfbeis, T. Blunk, M. B. Schulz, and A. Göpferich. Determination of oxygen gradients in engineered tissue using a fluorescent sensor. Biotechnology and bioengineering, 80(1):73-83, 2002.

[20] Y. Kim and H. G. Othmer. A hybrid model of tumor-stromal interactions in breast cancer. Bulletin of mathematical biology, 75(8):1304-1350, 2013.

[21] Y. Kim, M. Stolarska, and H. Othmer. The role of the microenvironment in tumor growth and invasion. Progress in Biophysics 8 Molecular Biology, 106, pages 353-379, 2011.

[22] F. Kleinhans. Membrane permeability modeling : Kedem-Katchalsky vs a two-parameter formalism. Cryobiology, 37 , pages 271-289, 1998.

[23] A. Köhrmann, U. Kammerer, M. Kapp, J. Dietl, and J. Anacker. Expression of matrix metalloproteinases (MMPs) in primary human breast cancer and breast cancer cell lines: new findings and review of the literature. BMC Cancer, 9:188, 2009.

[24] G. Lefebvre, F. Cornelis, P. Cumsille, T. Colin, C. Poignard, and O. Saut. Spatial modelling of tumour drug resistance: the case of gist liver metastases. Mathematical Medicine and Biology, 2016.

[25] M. Leguèbe, A. Silve, L. Mir, and C. Poignard. Conducting and permeable states of cell membrane submitted to high voltage pulses. Mathematical and numerical studies validated by the experiments. J. Theor. Biol., 360:83-94, 2014.

[26] P. Macklin, M. Edgerton, A. Thompson, and V. Cristini. Patient-calibrated agent-based modelling of ductal carcinoma in situ (DCIS) : from microscopic measurements to macroscopic predictions of clinical progression. $J$ Theor Biol., 301, pages 122-140, 2012.

[27] K. Norton, M. Wininger, G. Bhanot, S. Ganesan, N. Barnard, and T. Shinbrot. A 2D mechanistic model of breast ductal carcinoma in situ (DCIS) morphology and progression. J Theor Biol., 263, pages 393-406, 2010.

[28] C. Overall and C. López-Otín. Strategies for MMP inhibition in cancer: innovations for the post-trial era. Nat Rev Cancer, Vol. 2, pages 657-672, 2002.

[29] R. Perrussel and C. Poignard. Asymptotic expansion of steady-state potential in a high contrast medium with a thin resistive layer. Applied Mathematics and Computation, pages 48-65, 2013.

[30] R. Poincloux, F. Lizárraga, and P. Chavrier. Matrix invasion by tumour cells: a focus on mt1-mmp trafficking to invadopodia. Journal of Cell Science, 122(17):3015-3024, 2009.

[31] D. Ramsay, J. Kent, R. Hartmann, and P. Hartmann. Anatomy of the lactating human breast redefined with ultrasound imaging. J Anat., 206, pages 525-534, 2005.

[32] S. Rha, J. Kim, J. Roh, K. Lee, J. Min, B. Kim, and H. Chung. Sequential production and activation of matrixmetalloproteinase-9 (MMP-9) with breast cancer progression. Breast Cancer Researsh and Treatment, 43, pages 175-181, 1997.

[33] B. Ribba, O. Saut, T. Colin, D. Bresh, E. Grenier, and J.-P. Boissel. A multiscale mathematical model of avascular tumor growth to investigate the therapeutic benefit of anti-invasive agents. J Theor Biol., 243(4), pages 532-541, 2006.

[34] R. Sakr. Carcinomes canalaires in situ du sein : rôle potentiel de la biologie moléculaire. Gynécologie Obstétrique E Fertilité, 41, pages 45-53, 2012.

[35] J. S. Sartakhti, M. H. Manshaei, and M. Sadeghi. Mmp-timp interactions in cancer invasion: An evolutionary game-theoretical framework. Journal of Theoretical Biology, pages -, 2016.

[36] J. A. Sethian. Fast marching methods. SIAM review, 41(2):199-235, 1999.

[37] J. Tse, G. Cheng, J. Tyrrell, S. Wilcox-Adelman, Y. Boucher, R. Jain, and L. Munn. Mechanical compression drives cancer toward invasive phenotype. PNAS, Vol.109, No.3, pages 911-916, Jan 2012. 
[38] A. Waterston and M. Bower. TNF and cancer : good or bad ? Cancer Therapy, vol 2, pages 131-148, 2004.

[39] V. Weaver, A. Fisher, O. Peterson, and M. Bissell. The importance of the microenvironment in breast cancer progression : recapitulation of mammary tumorigenesis using a unique human mammary epithelial cell model and a three-dimensional culture assay. Biochem Cell Biol., 74, pages 833-851, 1996.

[40] S. Yang. To revert breast cancer cells, give them the squeeze. UC Berkeley News Center, Dec 17, 2012. http: //newscenter. berkeley.edu/

\section{APPENDIX: NUMERICAL PARAMETERS FOR THE SIMULATIONS}

The following table summarizes the values of the parameters used in the numerical simulations.

\begin{tabular}{|cl|}
\hline \multicolumn{2}{|c|}{ Core model } \\
\hline$k$ & 1 \\
$\beta_{P}$ & 20 \\
$\beta_{N}$ & 5 \\
$\Lambda$ & 30 \\
\hline
\end{tabular}

\begin{tabular}{|c|c|c|c|}
\hline \multicolumn{4}{|c|}{ Nutrients, TNF and MMPs } \\
\hline \multicolumn{2}{|c|}{ Nutrients } & \multicolumn{2}{|c|}{ MMPs } \\
\hline$\Theta_{P}$ & 0.6 & $d_{M^{T}}$ & $0.002 / 0.01$ \\
\hline$\Theta_{N}$ & 0.4 & $\gamma_{T}$ & 1 \\
\hline$\theta^{\max }$ & $0.8 / 1$ & $d_{M^{*}}$ & 0.01 \\
\hline$c$ & $0 / 2$ & $\gamma_{\star}$ & $0 / 1$ \\
\hline \multicolumn{2}{|c|}{ TNFs } & $M_{t h}$ & 0.5 \\
\hline$\tau_{P}$ & 0.3 & $\Pi_{t h}$ & 0.2 \\
\hline$\tau_{N}$ & 0.8 & $\gamma_{M}$ & $0 / 1$ \\
\hline$d_{\tau}$ & 0.01 & & \\
\hline$\gamma_{\tau}$ & $0 / 1$ & & \\
\hline
\end{tabular}

Table 1. Simulation parameters. Values are empirically determined and not calibrated. 